\title{
Interior and exterior sound field control using general two-dimensional first-order sources
}

\author{
M. A. Polettia \\ Industrial Research Ltd., P.O. Box 31-310, Lower Hutt, New Zealand \\ T. D. Abhayapala \\ School of Engineering, Australian National University College of Engineering and Computer Science, \\ The Australian National University, Canberra, Australia
}

(Received 8 August 2010; revised 28 October 2010; accepted 29 October 2010)

\begin{abstract}
Reproduction of a given sound field interior to a circular loudspeaker array without producing an undesirable exterior sound field is an unsolved problem over a broadband of frequencies. At low frequencies, by implementing the Kirchhoff-Helmholtz integral using a circular discrete array of linesource loudspeakers, a sound field can be recreated within the array and produce no exterior sound field, provided that the loudspeakers have azimuthal polar responses with variable first-order responses which are a combination of a two-dimensional (2D) monopole and a radially oriented 2D dipole. This paper examines the performance of circular discrete arrays of line-source loudspeakers which also include a tangential dipole, providing general variable-directivity responses in azimuth. It is shown that at low frequencies, the tangential dipoles are not required, but that near and above the Nyquist frequency, the tangential dipoles can both improve the interior accuracy and reduce the exterior sound field. The additional dipoles extend the useful range of the array by around an octave.
\end{abstract} (C) 2011 Acoustical Society of America. [DOI: 10.1121/1.3518772]

PACS number(s): 43.60.Tj, 43.55.Jz, 43.38.Md, 43.60.Sx [EJS]

Pages: 234-244

\section{INTRODUCTION}

Sound reproduction systems aim to produce the exact reconstruction of a desired sound field within a discrete array of loudspeakers. For three-dimensional (3D) sound fields this is possible using a regular array that fully encloses the reproduction region, up to the Nyquist frequency, where the loudspeakers are a half-wavelength apart. ${ }^{1-6}$ In the $2 \mathrm{D}$ case a circular array of line sources can reproduce sound fields with height invariance, and point sources can approximate this ideal in the plane of the array. $2,6,7$

When sound reproduction systems with point- or linemonopole sources are used in reverberant spaces, a desired interior sound field is produced within the array by the direct sound from the loudspeakers, but an unwanted exterior field is also produced that radiates outward from the array. This exterior sound field reflects from room boundaries and produces a reverberant field within the array interior. This reverberant field interferes with the desired direct sound field generated within the array, reducing the accuracy of the reproduced field. The reverberant field can be reduced by increasing the absorption of the room surfaces. Alternatively, the exterior field generated by the array can be reduced by using directional loudspeakers that radiate higher sound levels into the array interior than they do to the exterior of the array. For example, in Ref. 8 spherical arrays of first-order, fixed-directivity loudspeakers are used for sound reproduction with a reduced exterior field, and it is verified that the increase in direct to reverberant ratio is maximized for hyper-cardioid loudspeakers.

\footnotetext{
a) Author to whom correspondence should be addressed. Electronic mail: m.poletti@irl.cri.nz
}

If independently controllable monopole and dipole sources are used then the exterior sound field can be eliminated at frequencies below the Nyquist frequency of the array by implementing the Kirchhoff-Helmholtz $(\mathrm{K}-\mathrm{H})$ integral. $^{9-12}$ The dipole sources are oriented normal to the surface of the reproduction volume, and for a spherical loudspeaker array, they are radially oriented. If the monopole and dipole elements are combined in a single loudspeaker, the loudspeakers have radially oriented, variable first-order polar responses. The use of variable-directivity loudspeakers thus allows the elimination of low-frequency reverberant sound without the need for acoustic modification of the room and without the use of pre-compensation systems which can remove the reverberant field produced by arrays of monopole sources. ${ }^{13-18}$

The use of tangential dipoles orthogonal to the normal is not required in the $\mathrm{K}-\mathrm{H}$ integral, and these dipoles do not radiate into the center of the array. With a discrete loudspeaker array, the $\mathrm{K}-\mathrm{H}$ integral can only be approximated at low frequencies, and tangential dipoles may improve sound field reconstruction at positions away from the center of the array and reduce the exterior sound field. This paper therefore considers sound reproduction using general first-order loudspeakers which include tangential dipoles. For simplicity, 2D sound reproduction using a circular array of line sources, with first-order polar responses in azimuth, will be assumed, which allows the ideal reproduction of sound fields with height invariance.

We have two main aims in the paper. The first is to determine if general first-order sources can improve interior sound reproduction in comparison with the radial-only case. Second, we wish to investigate whether exterior sound field cancellation is improved by the use of tangential dipoles. We expect that at low frequencies, the tangential dipoles are 
of no benefit for exterior cancellation, but they may improve the exterior cancellation at frequencies above the Nyquist frequency of the array.

Section II of this paper introduces the cylindrical harmonic descriptions of first-order sources. Section III considers the 2D sound reproduction problem. We derive fundamental solutions for the circular array case based on a continuous distribution of sources ${ }^{3,6,19}$ and then implement these with sampled approximations. The $\mathrm{K}-\mathrm{H}$ integral and the fixed-directivity solutions for the 2D case are first derived, and then the solutions for arrays with radial-only and radial-plus-tangential dipoles are derived, both with and without the cancellation of the exterior field. In Sec. IV, numerical simulations are used to generate the sound fields for the derived solutions. The accuracy of reproduction within the array is quantified by the angle-averaged (radial) squared error. $^{2,8}$ The exterior sound is quantified by the angle-averaged squared magnitude of the sound pressure relative to that of the desired pressure at the loudspeaker radius. We present simulation results for one frequency below the Nyquist frequency and for one above it, and then quantify the average interior and exterior performance of the array solutions as a function of frequency.

While the theory uses line sources of infinite length with variable polar responses in azimuth, we will refer to these as loudspeakers. Practical loudspeakers more correctly approximate point sources, but infinite line sources can be approximated in rooms by vertical arrays of loudspeakers or by electrostatic speakers. $^{20}$

\section{THEORETICAL BACKGROUND}

\section{A. Cylindrical expansions of sound fields}

The spatial variation of a sound field expressed in cylindrical coordinates at a point $(r, \phi, z)$ within a region of space, at angular frequency $\omega$, can be written as ${ }^{21}$

$p(r, \phi, z, \omega)=\sum_{m=-\infty}^{\infty} e^{i m \phi} \int_{-\infty}^{\infty} A_{m}\left(k_{z}, \omega\right) J_{m}\left(k_{r} r\right) e^{i k_{z} z} d k_{z}$,

where $A_{m}\left(k_{z}, k\right)$ is the $m$ th coefficient function of the wavenumber $k$, with vertical component $k_{z}=\sqrt{k^{2}-k_{r}^{2}}, J_{m}(\bullet)$ is the cylindrical Bessel function of the first kind, and $\phi$ is the azimuthal angle measured from the $x$-axis. We will assume in what follows that the sound field is independent of $z, k_{r}=k$, and the sound field expansion therefore simplifies to

$$
p(r, \phi, k)=\sum_{m=-\infty}^{\infty} J_{m}(k r) A_{m}(k) e^{i m \phi} .
$$

Each term $\exp (\operatorname{im} \phi)$ in the expansion may be referred to as a phase mode. ${ }^{7}$ We are interested in the reproduction of this sound field using sound sources of infinite extent in $z$, which have first-order directivity in azimuth $\phi$. We will assume a time dependency of the form $\exp (-i \omega t)$.

\section{B. Description of first-order sources}

The field due to a line source parallel to the $z$-axis located at $\vec{r}_{s}=\left(r_{s}, \phi_{s}\right)$ has the general form, ${ }^{22}$

$$
\begin{aligned}
& p_{\text {line }}(r, \phi, k, t) \\
& \quad=e^{-i \omega t} H_{0}^{(1)}\left(k\left\|\vec{r}-\vec{r}_{s}\right\|\right) \\
& \quad=e^{-i \omega t}\left\{\begin{array}{ll}
\sum_{m=-\infty}^{\infty} J_{m}(k r) H_{m}^{(1)}\left(k r_{s}\right) e^{i m\left(\phi-\phi_{s}\right)}, & r<r_{s} \\
\sum_{m=-\infty}^{\infty} J_{m}\left(k r_{s}\right) H_{m}^{(1)}(k r) e^{i m\left(\phi-\phi_{s}\right)}, & r>r_{s}
\end{array},\right.
\end{aligned}
$$

where $H_{m}^{(1)}(x)$ is the cylindrical Hankel function of the first kind and $\left\|\vec{r}-\vec{r}_{s}\right\|$ is the norm of the vector $\vec{r}-\vec{r}_{s}$. We will equivalently refer to the line source as a $2 \mathrm{D}$ monopole as its response is independent of $z$.

The derivative with respect to the magnitude of $\vec{r}_{s}, r_{s}$ is the $2 \mathrm{D}$ radial dipole,

$$
\frac{\partial}{\partial r_{s}}\left\{H_{0}\left(k\left\|\vec{r}-\vec{r}_{s}\right\|\right)\right\}=k H_{0}^{\prime}\left(k\left\|\vec{r}-\vec{r}_{s}\right\|\right) \cos \phi_{d},
$$

where $H_{m}^{\prime}(\bullet)$ is the first derivative of $H_{m}(\bullet)$ and

$$
\cos \phi_{d}=\frac{r_{s}-r \cos \left(\phi-\phi_{s}\right)}{\left\|\vec{r}_{s}-\vec{r}\right\|}
$$

is the cosine of the angle between $\vec{r}_{s}$ and $\vec{r}$. The radial derivative can also be found from the cylindrical expansion in Eq. (3):

$$
\begin{aligned}
\frac{\partial}{\partial r_{s}}\left\{H_{0}^{(1)}\left(k\left\|\vec{r}-\vec{r}_{s}\right\|\right)\right\} \\
\quad=\left\{\begin{array}{cl}
k \sum_{m=-\infty}^{\infty} J_{m}(k r) H_{m}^{\prime}\left(k r_{s}\right) e^{i m\left(\phi-\phi_{s}\right)}, & r<r_{s} \\
k \sum_{m=-\infty}^{\infty} J_{m}^{\prime}\left(k r_{s}\right) H_{m}^{(1)}(k r) e^{i m\left(\phi-\phi_{s}\right)}, & r>r_{s}
\end{array} .\right.
\end{aligned}
$$

The dipole can be equalized so that its magnitude is similar to that of the line source by equalizing by $1 / i k{ }^{8}$ The equalized 2D dipole then has the expansion,

$$
\begin{aligned}
& p_{d, r_{s}}(r, \phi, k) \\
& =-i H_{0}^{\prime}\left(k\left\|\vec{r}-\vec{r}_{s}\right\|\right) \cos \phi_{d} \\
& =\left\{\begin{array}{ll}
-i \sum_{m=-\infty}^{\infty} J_{m}(k r) H_{m}^{\prime}\left(k r_{s}\right) e^{i m\left(\phi-\phi_{s}\right)}, & r<r_{s} \\
-i \sum_{m=-\infty}^{\infty} J_{m}^{\prime}\left(k r_{s}\right) H_{m}^{(1)}(k r) e^{i m\left(\phi-\phi_{s}\right)}, & r>r_{s}
\end{array} .\right.
\end{aligned}
$$

The sound field of a 2D dipole tangential to a circle at a distance $r_{s}$ from the origin has the same form as Eq. (4), with angle term $\sin \left(\phi_{d}\right)$. The cylindrical harmonic expansion of the tangential dipole may be found from the derivative of the line source Eq. (3) with respect to $\phi_{s}$ and the fact that $d p / d x$ $=d p /\left(r_{s} d \phi_{s}\right)$,

$$
\begin{aligned}
& \frac{1}{r_{s}} \frac{d p_{\text {line }}(r, \phi, k)}{d \phi_{s}} \\
& \quad= \begin{cases}\frac{-i}{r_{s}} \sum_{m=-\infty}^{\infty} m J_{m}(k r) H_{m}^{(1)}\left(k r_{s}\right) e^{i m\left(\phi-\phi_{s}\right)}, & r<r_{s} \\
\frac{-i}{r_{s}} \sum_{m=-\infty}^{\infty} m J_{m}\left(k r_{s}\right) H_{m}^{(1)}(k r) e^{i m\left(\phi-\phi_{s}\right)}, & r>r_{s}\end{cases}
\end{aligned}
$$


This may also be equalized by $i k$ to produce a response approximately the same as that of the line source,

$$
\begin{aligned}
p_{d, \phi_{s}}(r, \phi, k) & =i H_{0}^{\prime}\left(k\left\|\vec{r}-\vec{r}_{s}\right\|\right) \cos \theta_{d} \\
= & \left\{\begin{array}{ll}
\frac{-1}{k r_{s}} \sum_{m=-\infty}^{\infty} m J_{m}(k r) H_{m}\left(k r_{s}\right) e^{i m\left(\phi-\phi_{s}\right)}, & r<r_{s} \\
\frac{-1}{k r_{s}} \sum_{m=-\infty}^{\infty} m J_{m}\left(k r_{s}\right) H_{m}^{(1)}(k r) e^{i m\left(\phi-\phi_{s}\right)}, & r>r_{s}
\end{array},\right.
\end{aligned}
$$

where

$$
\cos \theta_{d}=\frac{r \cos \left(\phi-\left(\phi_{s}+\pi / 2\right)\right)}{\left\|\vec{r}_{s}-\vec{r}\right\|}
$$

From Eqs. (3), (7), and (9), a general 2D first-order source has the form

$$
p_{\alpha, \beta, \gamma}(r, \phi, k)= \begin{cases}\sum_{m=-\infty}^{\infty} J_{m}(k r)\left[\left(\alpha-\frac{m}{k r_{s}} \gamma\right) H_{m}\left(k r_{s}\right)-i \beta H_{m}^{\prime}\left(k r_{s}\right)\right] e^{i m\left(\phi-\phi_{s}\right)}, \quad r<r_{s} \\ \sum_{m=-\infty}^{\infty} H_{m}^{(1)}(k r)\left[\left(\alpha-\frac{m}{k r_{s}} \gamma\right) J_{m}\left(k r_{s}\right)-i \beta J_{m}^{\prime}\left(k r_{s}\right)\right] e^{i m\left(\phi-\phi_{s}\right)}, \quad r>r_{s}\end{cases}
$$

where $\alpha, \beta$, and $\gamma$ are the monopole, radial, and tangential dipole weighting coefficients, respectively. Two special cases may be defined from Eq. (11). First, a radial variable-directivity source is obtained by setting $\gamma=0$. Second, a radial, fixed-directivity source is obtained by setting $\gamma=0, \alpha=\delta$, and $\beta=(1-\delta)$, where $\delta$ is the first-order parameter, yielding

$$
p_{\delta}(r, \phi, k)=\left\{\begin{array}{ll}
\sum_{m=-\infty}^{\infty} J_{m}(k r)\left[\delta H_{m}\left(k r_{s}\right)-i(1-\delta) H_{m}^{\prime}\left(k r_{s}\right)\right] e^{i m\left(\phi-\phi_{s}\right)}, & r<r_{s} \\
\sum_{m=-\infty}^{\infty} H_{m}^{(1)}(k r)\left[\delta J_{m}\left(k r_{s}\right)-i(1-\delta) J_{m}^{\prime}\left(k r_{s}\right)\right] e^{i m\left(\phi-\phi_{s}\right)}, & r>r_{s}
\end{array} .\right.
$$

For $\delta=1$ this produces a 2D monopole (line) source, for $\delta=0$ it produces an (equalized) 2D dipole, and for $\delta=0.25$ a 2D hyper-cardioid far-field polar response is produced. ${ }^{8}$

\section{Sound field due to a continuous distribution of first-order sources}

In this paper we consider the generation of sound fields using a continuous circular distribution of first-order sources which allows sampling effects to be ignored. . $^{3,6,19}$ The source distribution is positioned at $\vec{r}_{v}=\left(r_{L}, \phi_{v}\right), \phi_{v} \in[0,2 \pi]$. The sound field is then the integral of Eq. (11) over source angles $\phi_{v}$,

$$
p(r, \phi, k)= \begin{cases}\sum_{m=-\infty}^{\infty} J_{m}(k r) \frac{e^{i m \phi}}{2 \pi} \int_{0}^{2 \pi}\left\{\left[\alpha\left(\phi_{v}\right)-\frac{m}{k r_{L}} \gamma\left(\phi_{v}\right)\right] H_{m}\left(k r_{L}\right)-i \beta\left(\phi_{v}\right) H_{m}^{\prime}\left(k r_{L}\right)\right\} e^{i m \phi_{v}} d \phi_{v}, & r<r_{L} \\ \sum_{m=-\infty}^{\infty} H_{m}(k r) \frac{e^{i m \phi}}{2 \pi} \int_{0}^{2 \pi}\left\{\left[\alpha\left(\phi_{v}\right)-\frac{m}{k r_{L}} \gamma\left(\phi_{v}\right)\right] J_{m}\left(k r_{L}\right)-i \beta\left(\phi_{v}\right) J_{m}^{\prime}\left(k r_{L}\right)\right\} e^{i m \phi_{v}} d \phi_{v}, & r>r_{L}\end{cases}
$$

where $\alpha\left(\phi_{v}\right), \beta\left(\phi_{v}\right)$, and $\gamma\left(\phi_{v}\right)$ are the amplitudes of the monopole, radial dipole, and tangential dipole components, respectively, as a function of angle.

We derive solutions for these amplitude weightings in Sec. III for a number of specific fixed- and variable-directivity arrays. We require the interior field for $r<r_{L}$ to equal the arbitrary interior field in Eq. (2) and may also require the exterior field for $r>r_{L}$ to be zero. The sampling requirements for implementation of the continuous solutions using a discrete circular array of sources ${ }^{7,23}$ will be discussed in Sec. III D. 


\section{SOUND REPRODUCTION USING CIRCULAR FIRST-ORDER LINE ARRAYS}

The required source amplitudes $\alpha\left(\phi_{v}\right), \beta\left(\phi_{v}\right)$, and $\gamma\left(\phi_{v}\right)$ will be derived by assuming that they are of the form,

$$
\begin{aligned}
& \alpha\left(\phi_{v}\right)=\sum_{m=-\infty}^{\infty} a_{m} e^{i m \phi_{v}}, \\
& \beta\left(\phi_{v}\right)=\sum_{m=-\infty}^{\infty} b_{m} e^{i m \phi_{v}},
\end{aligned}
$$

and

$$
\gamma\left(\phi_{v}\right)=\sum_{m=-\infty}^{\infty} g_{m} e^{i m \phi_{v}}
$$

and employing the orthonormality of the phase modes. This approach avoids the large matrix inversions that are used in the spherical case, where orthonormality of discrete samplings of spherical harmonics is not guaranteed. ${ }^{8}$ We first derive the source amplitudes for the $\mathrm{K}-\mathrm{H}$ integral (the ideal solution at low frequencies) and the fixed-directivity source (which we use for comparison in Sec. IV).

\section{A. $\mathrm{K}-\mathrm{H}$ integral formula}

The $\mathrm{K}-\mathrm{H}$ integral for a bounded volume $\Omega$ is of the form,

$p(\vec{r})=\int_{\Omega}\left[\frac{\partial p\left(\vec{r}_{v}\right)}{\partial \vec{n}\left(\vec{r}_{v}\right)} G\left(\vec{r} \mid \vec{r}_{v}\right)-i k p\left(\vec{r}_{v}\right) \frac{1}{i k} \frac{\partial G\left(\vec{r} \mid \vec{r}_{v}\right)}{\partial \vec{n}\left(\vec{r}_{v}\right)}\right] d \Omega$,

where $\vec{r}_{v}$ is a vector which lies on the surface $\Omega$ and $\vec{n}\left(\vec{r}_{v}\right)$ is the outward-facing normal from $\Omega$. In the $2 \mathrm{D}$ case, we assume a 2D Green function of the form $G\left(\vec{r} \mid \vec{r}_{v}\right)$ $=H_{0}\left(k\left\|\vec{r}-\vec{r}_{v}\right\|\right)$. (We ignore the $i / 4$ scale factor for convenience. ${ }^{19,21}$ )

For the case of an infinitely tall cylinder of radius $r_{L}$ and for a sound field of the form of Eq. (2), it can be shown that the $\mathrm{K}-\mathrm{H}$ integral is of the form,

$$
\begin{aligned}
p(r, \phi)= & \frac{i \pi r_{L}}{2} \int_{0}^{2 \pi}\left[\frac{\partial p\left(r_{L}, \phi_{v}\right)}{\partial r} H_{0}\left(k\left\|\vec{r}-\vec{r}_{v}\right\|\right)\right. \\
& \left.-i k p\left(r_{L}, \phi_{v}\right) \frac{1}{i k} \frac{\partial}{\partial r} H_{0}\left(k\left\|\vec{r}-\vec{r}_{v}\right\|\right)\right] d \phi_{v},
\end{aligned}
$$

where the $i k$ equalization of the dipole resulting from the derivative of the Green's function is explicitly included. This equation can be verified by substituting from Eqs. (2) and (3) and employing the Wronskian expression

$$
J_{m}(x) H_{m}^{\prime}(x)-J_{m}^{\prime}(x) H_{m}(x)=\frac{2 i}{\pi x} .
$$

The monopole amplitude is, from Eq. (2),

$$
\alpha\left(\phi_{v}\right)=\frac{i \pi k r_{L}}{2} \sum_{m=-\infty}^{\infty} J_{m}^{\prime}\left(k r_{L}\right) A_{m} e^{i m \phi_{s}},
$$

and the (equalized) radial dipole amplitude is

$$
\beta\left(\phi_{v}\right)=\frac{\pi k r_{L}}{2} \sum_{m=-\infty}^{\infty} J_{m}\left(k r_{L}\right) A_{m} e^{i m \phi_{s}} .
$$

\section{B. Fixed-directivity radial sources}

Consider the field due to a continuous circular array of $L$ first-order, radially oriented sources, Eq. (13), for which $\gamma\left(\phi_{v}\right)=0, \alpha\left(\phi_{v}\right)=\delta$, and $\beta\left(\phi_{v}\right)=1-\delta$. These sources produce an inward-facing cardioid for $\delta=0.5$ and a hyper-cardioid for $\delta=0.25 .^{8}$ Assume that the source amplitude is

$$
\chi\left(\phi_{v}\right)=\sum_{m=-\infty}^{\infty} c_{m} e^{i m \phi_{v}}
$$

Substituting this and the cylindrical expansion in Eq. (12) into Eq. (13) and employing the orthogonality of the phase modes yields

$$
c_{m}=\frac{A_{m}}{\delta H_{m}\left(k r_{L}\right)-i(1-\delta) H_{m}^{\prime}\left(k r_{L}\right)} .
$$

The loudspeaker amplitudes are then given by

$$
\chi\left(\phi_{v}\right)=\sum_{m=-\infty}^{\infty} \frac{A_{m}}{\delta H_{m}\left(k r_{L}\right)-i(1-\delta) H_{m}^{\prime}\left(k r_{L}\right)} e^{i m \phi_{v}} .
$$

\section{Variable-directivity sources}

We now consider the case of a continuous distribution of general first-order sources with expansions given in Eq. (11). We derive solutions with and without tangential dipoles and with or without the control of the exterior sound field.

\section{Interior/exterior solution for general 2D first-order sources}

The field produced by a continuous array [Eq. (13)] is required to produce the field in Eq. (2). Hence, for each $m$

$$
\begin{aligned}
\frac{1}{2 \pi} \int_{0}^{2 \pi}\left[\left(\alpha\left(\phi_{v}\right)-\frac{m}{k r_{L}} \gamma\left(\phi_{v}\right)\right) H_{m}\left(k r_{L}\right)\right. \\
\\
\left.\quad-i \beta\left(\phi_{v}\right) H_{m}^{\prime}\left(k r_{L}\right)\right] e^{-i m \phi_{v}} d \phi_{v}=A_{m}
\end{aligned}
$$

and for cancellation of the exterior field

$$
\begin{aligned}
\frac{1}{2 \pi} \int_{0}^{2 \pi}\left[\left(\alpha\left(\phi_{v}\right)-\frac{m}{k r_{L}}\right.\right. & \left.\gamma\left(\phi_{v}\right)\right) J_{m}\left(k r_{L}\right) \\
& \left.-i \beta\left(\phi_{v}\right) J_{m}^{\prime}\left(k r_{L}\right)\right] e^{-i m \phi_{v}} d \phi_{v}=0 .
\end{aligned}
$$

Substituting Eqs. (14) to (16) yields the general phase mode equations 


$$
\left[a_{m}-\frac{m}{k r_{L}} g_{m}\right] H_{m}\left(k r_{L}\right)-i b_{m} H_{m}^{\prime}\left(k r_{L}\right)=A_{m}
$$

and

$$
\left[a_{m}-\frac{m}{k r_{L}} g_{m}\right] J_{m}\left(k r_{L}\right)-i b_{m} J_{m}^{\prime}\left(k r_{L}\right)=0 .
$$

There are an infinite number of solutions to these two equations. The solution with the minimum energy is obtained by writing Eqs. (27) and (28) in matrix form

$$
\begin{aligned}
& {\left[\begin{array}{ccc}
H_{m}\left(k r_{L}\right) & \frac{-m}{k r_{L}} H_{m}\left(k r_{L}\right) & -i H_{m}^{\prime}\left(k r_{L}\right) \\
J_{m}\left(k r_{L}\right) & \frac{-m}{k r_{L}} J_{m}\left(k r_{L}\right) & -i J_{m}^{\prime}\left(k r_{L}\right)
\end{array}\right]\left[\begin{array}{l}
a_{m} \\
g_{m} \\
b_{m}
\end{array}\right]} \\
& =\boldsymbol{\Phi}\left[\begin{array}{l}
a_{m} \\
g_{m} \\
b_{m}
\end{array}\right]=\left[\begin{array}{c}
A_{m} \\
0
\end{array}\right]
\end{aligned}
$$

from which the minimum energy solution is ${ }^{2,24}$

$$
\left[\begin{array}{l}
a_{m} \\
g_{m} \\
b_{m}
\end{array}\right]=\boldsymbol{\Phi}^{H}\left[\boldsymbol{\Phi} \boldsymbol{\Phi}^{H}+\lambda \mathbf{I}_{2}\right]^{-1}\left[\begin{array}{c}
A_{m} \\
0
\end{array}\right]
$$

where $\lambda$ is regularization term added because the solution can be poorly conditioned at low frequencies and where $\mathbf{I}_{2}$ is the $2 \times 2$ identity matrix.

\section{Interior/exterior solution with radial first-order sources}

If we restrict the loudspeaker array to radial dipoles only, the solution for the weights may be found from Eqs. (27) and (28) with $g_{m}=0$. The mode equations in matrix form are

$$
\left[\begin{array}{cc}
H_{m}\left(k r_{L}\right) & -i H_{m}^{\prime}\left(k r_{L}\right) \\
J_{m}\left(k r_{L}\right) & -i J_{m}^{\prime}\left(k r_{L}\right)
\end{array}\right]\left[\begin{array}{l}
a_{m} \\
b_{m}
\end{array}\right]=\left[\begin{array}{c}
A_{m} \\
0
\end{array}\right]
$$

This may be solved directly using the Wronskian relation Eq. (19). The solution is the $\mathrm{K}-\mathrm{H}$ solution in Eqs. (20) and (21), as expected.

\section{Interior solution with general first-order sources}

If we allow general first-order sources and require the array to generate an interior field with no exterior constraint, then the single mode-matching equation is

$$
\left[\begin{array}{lll}
H_{m}\left(k r_{L}\right) & \frac{-m}{k r_{L}} H_{m}\left(k r_{L}\right) & -i H_{m}^{\prime}\left(k r_{L}\right)
\end{array}\right]\left[\begin{array}{l}
a_{m} \\
g_{m} \\
b_{m}
\end{array}\right]=A_{m} .
$$

The minimum energy solution is

$$
\begin{aligned}
& a_{m}= \frac{H_{m}^{*}\left(k r_{L}\right) A_{m}}{\left|H_{m}\left(k r_{L}\right)\right|^{2}\left(1+\frac{m^{2}}{\left(k r_{L}\right)^{2}}\right)+\left|H_{m}^{\prime}\left(k r_{L}\right)\right|^{2}}, \\
& g_{m}=-\frac{m}{k r_{L}} H_{m}^{*}\left(k r_{L}\right) A_{m} \\
&\left|H_{m}\left(k r_{L}\right)\right|^{2}\left(1+\frac{m^{2}}{\left(k r_{L}\right)^{2}}\right)+\left|H_{m}^{\prime}\left(k r_{L}\right)\right|^{2}
\end{aligned}
$$

and

$$
b_{m}=\frac{-i\left[H_{m}^{\prime}\left(k r_{L}\right)\right]^{*} A_{m}}{\left|H_{m}\left(k r_{L}\right)\right|^{2}\left(1+\frac{m^{2}}{\left(k r_{L}\right)^{2}}\right)+\left|H_{m}^{\prime}\left(k r_{L}\right)\right|^{2}}
$$

\section{Interior solution with radial first-order sources} is

With $\gamma\left(\phi_{v}\right)=0$, the mode equation for the interior field

$$
a_{m} H_{m}\left(k r_{L}\right)-i b_{m} H_{m}^{\prime}\left(k r_{L}\right)=A_{m}
$$

The minimum energy solution is

$$
a_{m}=\frac{H_{m}^{*}\left(k r_{L}\right) A_{m}}{\left|H_{m}\left(k r_{L}\right)\right|^{2}+\left|H_{m}^{\prime}\left(k r_{L}\right)\right|^{2}}
$$

and

$$
b_{m}=\frac{i\left[H_{m}^{\prime}\left(k r_{L}\right)\right]^{*} A_{m}}{\left|H_{m}\left(k r_{L}\right)\right|^{2}+\left|H_{m}^{\prime}\left(k r_{L}\right)\right|^{2}} .
$$

\section{Discrete circular arrays}

The results derived above assume a continuous array. The use of a discrete array introduces sampling-related errors into the reproduction of the sound field and to the approximation

$$
\hat{p}(r, \phi, k)=\left\{\begin{array}{l}
\sum_{m=-\infty}^{\infty} J_{m}(k r) \frac{e^{i m \phi}}{L} \sum_{l=1}^{L}\left\{\left[\alpha\left(\phi_{l}\right)-\frac{m}{k r_{L}} \gamma\left(\phi_{l}\right)\right] H_{m}\left(k r_{L}\right)-i \beta\left(\phi_{l}\right) H_{m}^{\prime}\left(k r_{L}\right)\right\} e^{i m \phi_{l},} \quad r<r_{L} \\
\sum_{m=-\infty}^{\infty} H_{m}(k r) \frac{e^{i m \phi}}{L} \sum_{l=1}^{L}\left\{\left[\alpha\left(\phi_{l}\right)-\frac{m}{k r_{L}} \gamma\left(\phi_{l}\right)\right] J_{m}\left(k r_{L}\right)-i \beta\left(\phi_{l}\right) J_{m}^{\prime}\left(k r_{L}\right)\right\} e^{i m \phi_{l},} \quad r>r_{L}
\end{array}\right.
$$


of the continuous source sound field $p(r, \phi, k)$ in Eq. (13). The array performance will equal that of the continuous array below what we will term the Nyquist frequency, in reference to the temporal sampling theorem. ${ }^{25}$ For a regular circular array of $L$ loudspeakers at radius $r_{L}$, the loudspeakers are positioned at angles $\phi_{l}=2 \pi l / L, l \in[0, L-1]$. The distance between loudspeakers is $d_{L}=\sqrt{2} r_{L} \sqrt{1-\cos (2 \pi / L)}$. Setting this to a half-wavelength yields the "Nyquist" frequency for the array

$$
f_{N}=\frac{c}{2 \sqrt{2} r_{L} \sqrt{1-\cos (2 \pi / L)}} \approx \frac{c L}{4 \pi r_{L}},
$$

where the approximation is valid for large $L$. This analysis defines the half-wavelength frequency, but it does not prove that the half-wavelength frequency is the frequency above which the circular array ceases to operate as the continuous case. This proof is obtained from the required order of the cylindrical expansion of the sound field Eq. (2). ${ }^{26}$ It has been shown that for a given $k r$, Eq. (2) can be truncated to an order $M=\lceil e \pi r / \lambda\rceil \approx\lceil k r\rceil$, where $e=\exp (1)=2.718$, and that the dimensionality of the $2 \mathrm{D}$ sound field is

$$
D_{r}^{2 \mathrm{D}}=2\left\lceil\frac{e \pi r}{\lambda}\right\rceil+1=2 M+1
$$

Since the order $M$ expansion in Eq. (2) has $2 M+1$ terms, then $L=D_{r}^{2 \mathrm{D}}=2 M+1$ loudspeakers will be sufficient to control these phase modes, and for a given $L$, the corresponding frequency is

$$
f_{D}=\frac{c(L-1)}{2 e \pi r_{L}} \approx f_{N}
$$

where the approximation is obtained for $e \approx 2$ and $L$ large. Hence, the half-wavelength frequency is approximately equal to the exact frequency required for accurate reproduction of the wavelength over the interior of the array.

More generally, Eq. (41) shows that the sound-field dimensionality varies with radius. For smaller radii, the number of loudspeakers required for accurate reproduction is smaller. Hence, for a given array the reproduction of a desired sound field will be possible up to a radius $r_{M}$,

$$
r_{M} \approx \frac{(L-1)}{2 k}
$$

beyond which the dimensionality of the sound field exceeds the number of loudspeakers. The Nyquist frequency in Eq. (40) ensures accuracy out to approximately the loudspeaker radius, and it also ensures the accurate synthesis of the exterior sound field. ${ }^{8}$
For the discrete array, the loudspeaker weights will be truncated to order $M=(L-1) / 2$ ( $L$ odd) to satisfy the sampling requirements discussed above. Hence, for example, the general monopole amplitude will be

$$
\alpha\left(\phi_{l}\right)=\frac{1}{L} \sum_{m=-M}^{M} a_{m} e^{i m \phi_{l}}
$$

and similarly for the other loudspeaker weights.

\section{SIMULATION RESULTS}

We now consider the performance of discrete approximations to the four solutions derived in Sec. III C, as shown in Table I, and compare their angle-averaged performance with that of the discrete fixed-directivity hyper-cardioid solution obtained from Eq. (24). We assume a circular array of $L=23$ first-order loudspeaker sources, at a radius of $r_{L}$ $=1.5 \mathrm{~m}$, which allows a maximum mode order of $M=11$ and produces a Nyquist frequency of $400 \mathrm{~Hz}$. We calculate the 11th order truncated solutions derived in Sec. III at the discrete loudspeaker angles, $\phi_{l}$, and generate the sound field using the direct form of the first-order sources (in terms of zeroth-order Bessel functions) from Eqs. (3), (7), and (9).

We will refer to the array using 2D monopole and radial dipole sources as the radial array, and the array using 2D monopole, radial dipole, and tangential dipoles as the radialplus-tangential array in what follows.

\section{A. Interior reproduction error}

The accuracy of the interior sound field reproduction with radius may be quantified by the angle-averaged relative error, 2,3

$$
\bar{\varepsilon}(k r)=\frac{\int_{0}^{2 \pi}|p(r, \phi, k)-\hat{p}(r, \phi, k)|^{2} d \phi}{\int_{0}^{2 \pi}|p(r, \phi, k)|^{2} d \phi} .
$$

The mean of this quantity over the range $r=\left[0, r_{I}\right], r_{I}<r_{L}$ will give a single figure of merit $\bar{\varepsilon}_{r_{I}}$ for the reproduction accuracy. Substituting from Eqs. (2) and (39) and employing the orthonormality of the phase modes yields

$$
\bar{\varepsilon}(k r)=\frac{\sum_{m=-\infty}^{\infty} J_{m}^{2}(k r)\left|A_{m}(k)-Z_{I}(m)\right|^{2}}{\sum_{m=-\infty}^{\infty} J_{m}^{2}(k r)\left|A_{m}(k)\right|^{2}}
$$

TABLE I. Simulation types.

\begin{tabular}{lll}
\hline \hline Simulation & \multicolumn{1}{c}{ 2D source configuration } & \multicolumn{1}{c}{ Control } \\
\hline 1 & Monopole + radial dipole & Interior \\
2 & Monopole + radial dipole & Interior + exterior cancellation \\
3 & Monopole + radial + tangential dipole & Interior \\
4 & Monopole + radial + tangential dipole & Interior + exterior cancellation \\
\hline \hline
\end{tabular}


where

$$
\begin{aligned}
Z_{I}(m)= & \sum_{l=1}^{L}\left[\left(\alpha\left(\phi_{l}\right)-\frac{m}{k r_{L}} \gamma\left(\phi_{l}\right)\right) H_{m}\left(k r_{L}\right)\right. \\
& \left.-i \beta\left(\phi_{l}\right) H_{m}^{\prime}\left(k r_{L}\right)\right] e^{-i m \phi_{l}} .
\end{aligned}
$$

\section{B. Exterior sound level}

The exterior cancellation may be quantified by the average over angle of the magnitude squared of the approximate sound pressure $\hat{p}(r, \phi, k)$, relative to the average magnitude squared pressure due to the desired sound field $p(r, \phi, k)$ at the loudspeaker radius $r_{L}$ :

$$
\bar{\Gamma}(k r)=\frac{\int_{0}^{2 \pi}|\hat{p}(r, \phi, k)|^{2} d \phi}{\int_{0}^{2 \pi}\left|p\left(r_{L}, \phi, k\right)\right|^{2} d \phi}, \quad r>r_{L} .
$$

Substituting from Eqs. (2) and (39) yields

$$
\bar{\Gamma}(k r)=\frac{\sum_{m=-\infty}^{\infty}\left|H_{m}(k r)\right|^{2}\left|Z_{E}(m)\right|^{2}}{\sum_{m=-\infty}^{\infty}\left|A_{m}(k)\right|^{2} J_{m}^{2}\left(k r_{L}\right)},
$$

where

$$
\begin{aligned}
Z_{E}(m)= & \sum_{l=1}^{L}\left[\left(\alpha\left(\phi_{l}\right)-\frac{m}{k r_{L}} \gamma\left(\phi_{l}\right)\right) J_{m}\left(k r_{L}\right)\right. \\
& \left.-i \beta\left(\phi_{l}\right) J_{m}^{\prime}\left(k r_{L}\right)\right] e^{-i m \phi_{l}} .
\end{aligned}
$$

The mean of this quantity over the range $r=\left[r_{E}, r_{\max }\right], r_{\max }$ $>r_{E}>r_{L}$ will give a single figure of merit $\bar{\Gamma}_{r_{E}, r_{\max }}$ for the relative exterior squared magnitude.

\section{Results}

Figures 1 and 2 show the sound fields produced by the radial array, with and without exterior cancellation, at $t=0$ for a line source at $x_{s}=3 \mathrm{~m}$ and at a frequency of $200 \mathrm{~Hz}$, below the Nyquist frequency of the array $(400 \mathrm{~Hz})$. The plots for the corresponding radial-plus-tangential array were essentially identical and are therefore not shown. The farfield polar responses of the sources are also shown centered on each corresponding source, and the maximum radius for accurate reproduction $r_{M}$ from Eq. (42) is shown as a dashed circle. For frequencies below the Nyquist frequency, this circle is at a radius $r_{M}>r_{L}$ and reproduction is accurate over the interior of the array.

Figure 1 shows the field without exterior cancellation. The generated wave propagates out of the array to the left, and a residual wave also propagates back toward the source. However, the directional element patterns reduce the amplitude of the upstream wave compared to what would be produced by omnidirectional sources. The loudspeakers on the left of the array produce essentially no output.

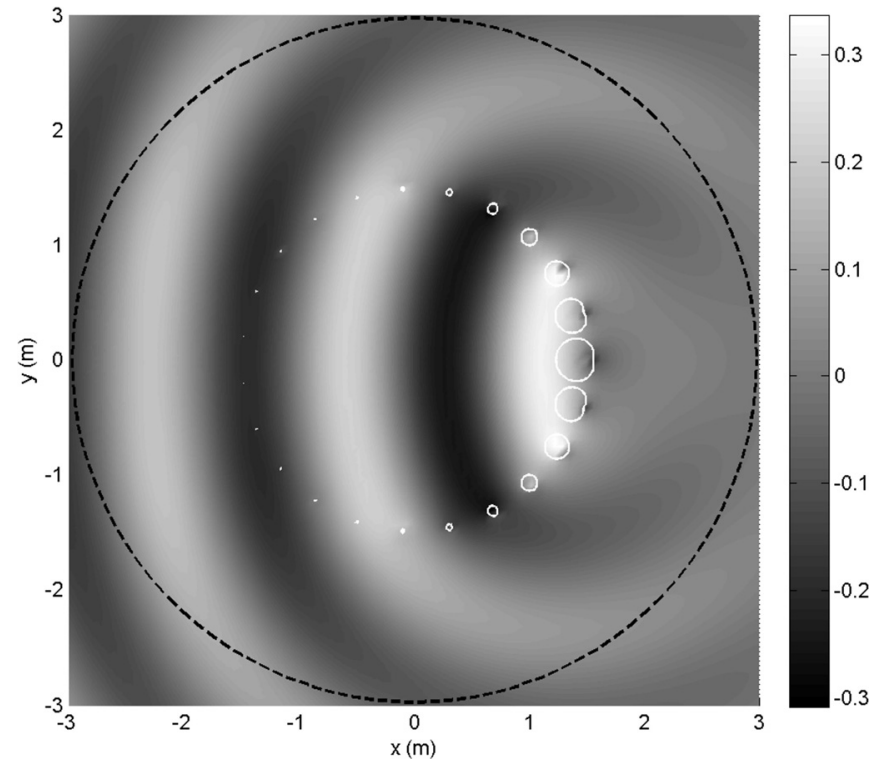

FIG. 1. Sound field at $t=0$ for a radial array of $L=23$ loudspeakers, $f=200 \mathrm{~Hz}$, and $r_{L}=1.5 \mathrm{~m}$, interior control only.

Figure 2 shows the field with exterior cancellation. The loudspeakers on the left hand side of the array now produce cardioid-like polar responses that emit a canceling wave field to eliminate the interior field that leaves the array on the "downstream" side. The loudspeakers lateral to the direction of propagation produce lower-amplitude responses which cancel the lateral wave leaving the array at angles in the region of $90^{\circ}$ and $270^{\circ}$. Those loudspeakers closer to the source angle $\left(0^{\circ}\right)$ also produce some interior field which assist in producing the desired field.

Figure 3 shows the interior reproduction error and exterior sound level for all four cases, together with the result for the fixed-directivity array with $\delta=0.25$ (hyper-cardioid responses). The interior error for the radial and radial-plustangential arrays at $1.3 \mathrm{~m}$ without exterior cancellation are 4 and $8 \mathrm{~dB}$ lower than the errors with exterior cancellation,

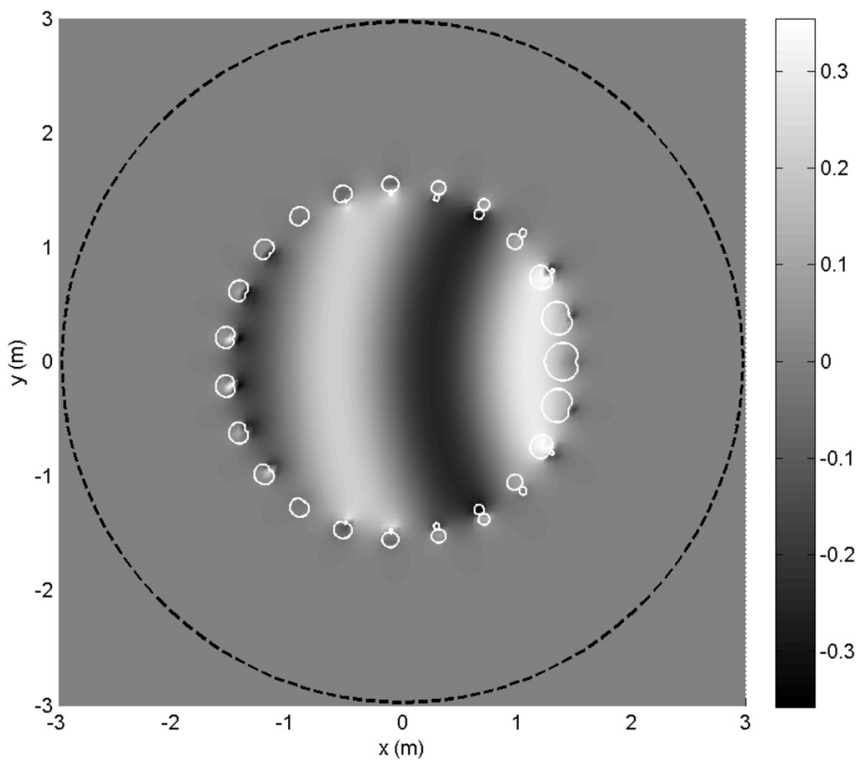

FIG. 2. Sound field at $t=0$ for a radial array of $L=23$ loudspeakers with exterior cancellation (K-H solution), $f=200 \mathrm{~Hz}$, and $r_{L}=1.5 \mathrm{~m}$. 


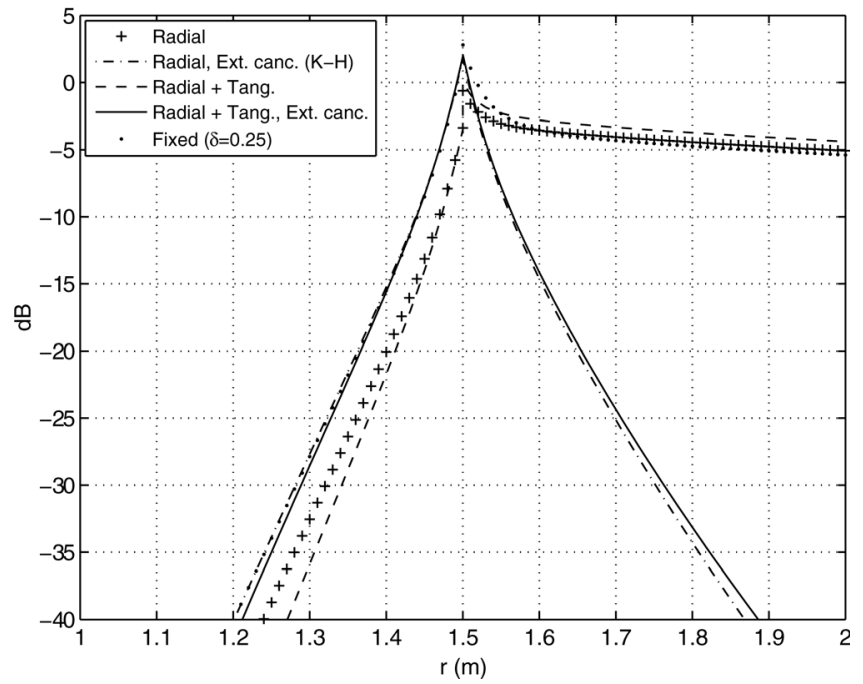

FIG. 3. Interior reproduction errors and exterior relative levels for $L=23$ loudspeaker arrays, $f=200 \mathrm{~Hz}$, and $r_{L}=1.5 \mathrm{~m}$.

respectively. Hence, the use of radial or radial-plus-tangential arrays produces a reproduction error similar to that of an array with more loudspeakers. Specifically, the error with $L=27$ fixed-directivity loudspeakers produces an interior error about the same as the radial array with 23 loudspeakers at a radius of $1.3 \mathrm{~m}$, and 29 fixed-directivity loudspeakers are required to produce an error as low as the radial-plus-tangential array at $1.3 \mathrm{~m}$. With the additional requirement for exterior cancellation, however, the improvement is lost.

The radial and radial-plus-tangential arrays with exterior cancellation produce similar low levels of exterior sound field, with the radial $(\mathrm{K}-\mathrm{H})$ array result around $1 \mathrm{~dB}$ lower than the radial-plus-tangential array field level at $1.8 \mathrm{~m}$.

The wave fields for the four simulations types at 600 $\mathrm{Hz}$, above the Nyquist frequency of the arrays $(400 \mathrm{~Hz})$, are shown in Figs. 4-7. Figure 4 shows the wave field for the

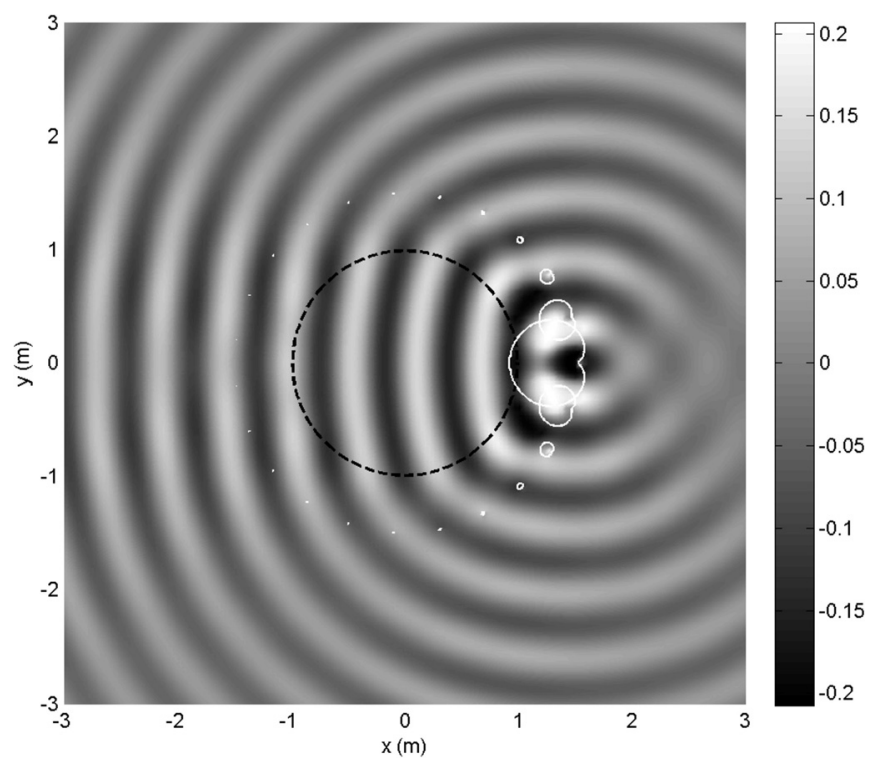

FIG. 4. Sound field at $t=0$ for a radial array of $L=23$ loudspeakers, $f=600 \mathrm{~Hz}$, and $r_{L}=1.5 \mathrm{~m}$, interior control only. The array Nyquist frequency is $400 \mathrm{~Hz}$.

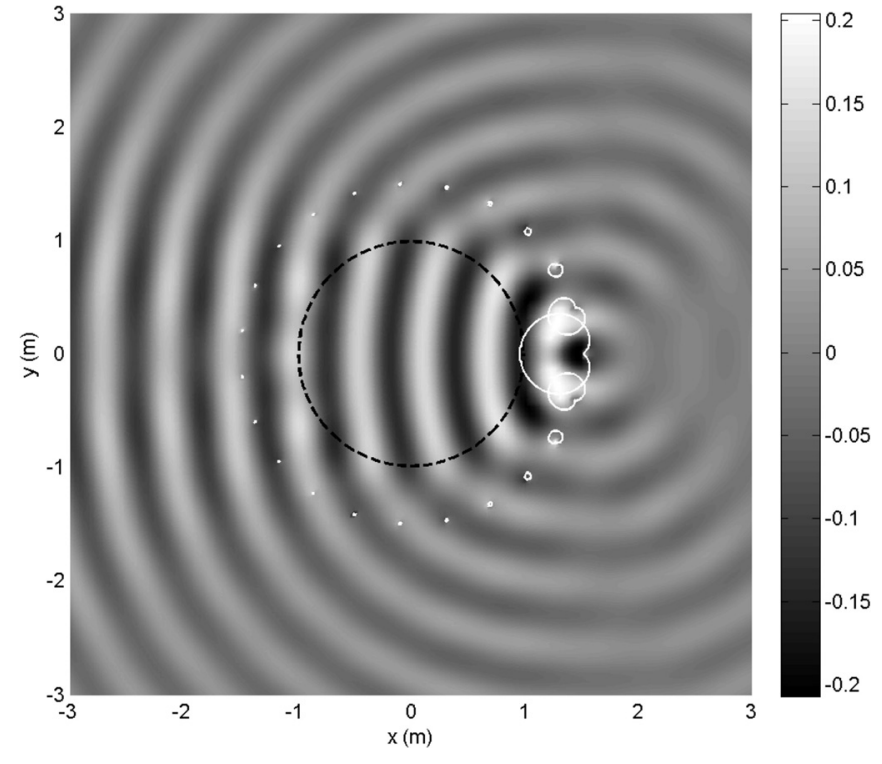

FIG. 5. Sound field at $t=0$ for a radial-plus-tangential array of $L=23$ loudspeakers, $f=600 \mathrm{~Hz}$, and $r_{L}=1.5 \mathrm{~m}$, interior control only. The array Nyquist frequency is $400 \mathrm{~Hz}$.

radial array and Fig. 5 the field for the radial-plus-tangential array, without exterior cancellation. The radial-plus-tangential array produces a smaller exterior field level, even though this was not a requirement on the solution. It can be seen that the two loudspeakers on each side of the loudspeaker at $0^{\circ}$ emitting the predominant sound are rotated in toward it, compared to Fig. 4, so that the nulls contribute to a reduced sound field in the angular regions at $-30^{\circ}$ and $+30^{\circ}$. The next two adjacent loudspeakers also produce rotated patterns compared to those in Fig. 4. This ability to rotate the pattern allows the general array to generate a more "compact" sound field with lower exterior field at high frequencies.

The fields with exterior cancellation are shown in Figs. 6 and 7 . The radial array is still able to cancel the downstream

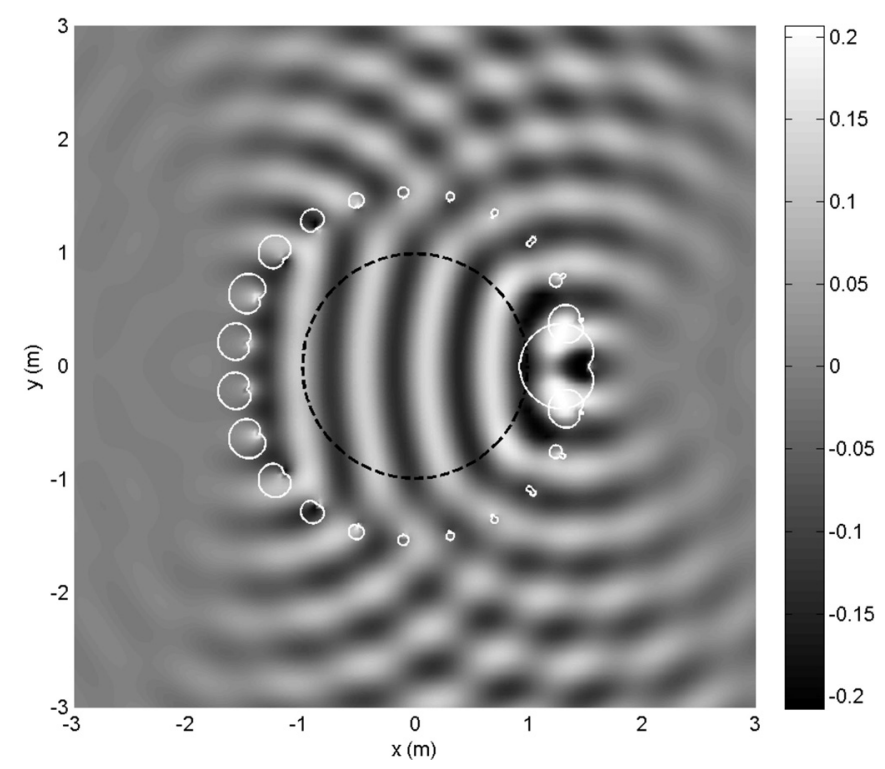

FIG. 6. Sound field at $t=0$ for a radial array of $L=23$ loudspeakers with exterior cancellation (K-H solution), $f=600 \mathrm{~Hz}$, and $r_{L}=1.5 \mathrm{~m}$. The array Nyquist frequency is $400 \mathrm{~Hz}$. 


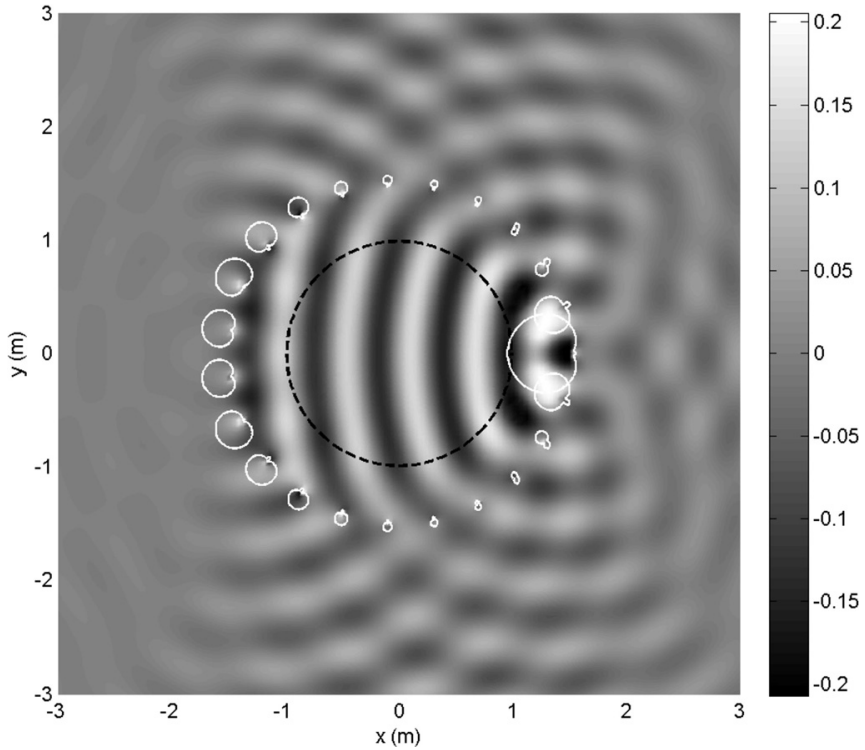

FIG. 7. Sound field at $t=0$ for a radial-plus-tangential array of $L=23$ loudspeakers with exterior cancellation, $f=600 \mathrm{~Hz}$, and $r_{L}=1.5 \mathrm{~m}$. The array Nyquist frequency is $400 \mathrm{~Hz}$.

field leaving the array and prevent the emission of sound toward the source but is unable to control the propagation of sound in lateral directions. The radial-plus-tangential array is better able to reduce this lateral sound emission and clearly shows a reduced exterior field in comparison with Fig. 6. However there is some increase in the sound traveling back toward the source.

Figure 8 shows the interior errors and exterior levels for the arrays. The interior errors are essentially the same for all solutions. With the additional requirement for exterior cancellation, the radial-plus-tangential array produces an error of around 3 to $4 \mathrm{~dB}$ lower than the non-cancelling solutions, and the radial-plus-tangential solution without exterior cancellation is also about $1 \mathrm{~dB}$ lower. Hence, the use of tangential dipoles can significantly reduce exterior sound when operating above the Nyquist frequency.

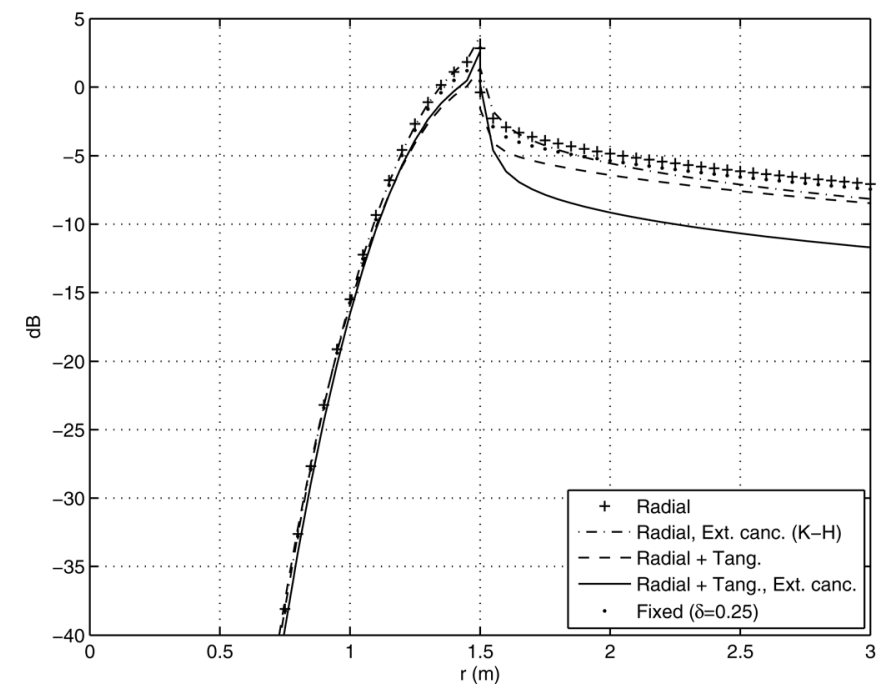

FIG. 8. Interior reproduction errors and exterior relative levels for $L=23$ loudspeaker arrays, $f=600 \mathrm{~Hz}$ and $r_{L}=1.5 \mathrm{~m}$.

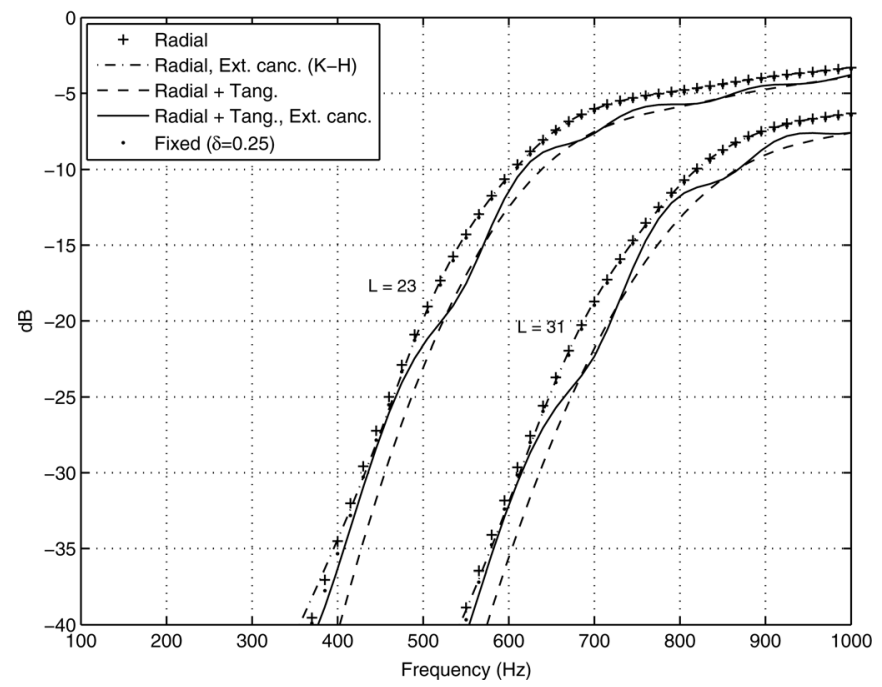

FIG. 9. Mean interior reproduction error from $r=0 \mathrm{~m}$ to $r=1.2 \mathrm{~m}$, versus frequency, for an $L=23$ and an $L=31$ loudspeaker array, $r_{L}=1.5 \mathrm{~m}$.

The mean interior errors over radii from 0 to $1.2 \mathrm{~m}$ as a function of frequency are shown in Fig. 9. The radial arrays and fixed-directivity array produce about the same error. However, the radial-plus-tangential array without exterior cancellation produces around $3 \mathrm{~dB}$ lower reproduction error than the radial and fixed-directivity arrays. With exterior cancellation, the radial-plus-tangential array error is about the same as the radial and fixed-directivity arrays below the Nyquist frequency, but for frequencies above the Nyquist frequency, the error oscillates with frequency between the fixed-directivity and radial-plus-tangential arrays without cancellation cases. This variation may be due to the fact that at high frequencies exterior cancellation increases the error produced on the far side of the array from the sound source, where the loudspeakers attempt to cancel the sound field leaving the array interior, and this error appears to fluctuate slightly with frequency. A detailed explanation of this effect is currently unknown.

Also shown in Fig. 9 is the interior error for $L=31$ loudspeakers at the same radius, producing a Nyquist frequency of $540 \mathrm{~Hz}$. The error performance is similar to the $L=23$ case but is shifted to higher frequencies. Hence performance scales with the array density as expected from Eq. (40).

The average exterior sound levels over radii from 1.8 to $3 \mathrm{~m}$ are shown in Fig. 10. The radial array without exterior cancellation produces about the same level as the fixed-directivity array. The radial-plus-tangential array produces around $1 \mathrm{~dB}$ higher level than the fixed-directivity array below Nyquist, but this reduces to about $2 \mathrm{~dB}$ below it at $550 \mathrm{~Hz}$, then slowly rises at higher frequencies. The radial array with cancellation ( $\mathrm{K}-\mathrm{H}$ solution) is able to cancel the exterior field up to the Nyquist frequency $(400 \mathrm{~Hz})$, where the level is $14 \mathrm{~dB}$ below the fixed-directivity level, but by $600 \mathrm{~Hz}$ there is no benefit to attempting exterior cancellation. However, the radial-plus-tangential array is able to maintain some cancellation relative to the fixed-directivity case to around 800 $\mathrm{Hz}$, twice the Nyquist frequency. The exterior level reduces above the Nyquist frequency to a minimum at $500 \mathrm{~Hz}$. The 


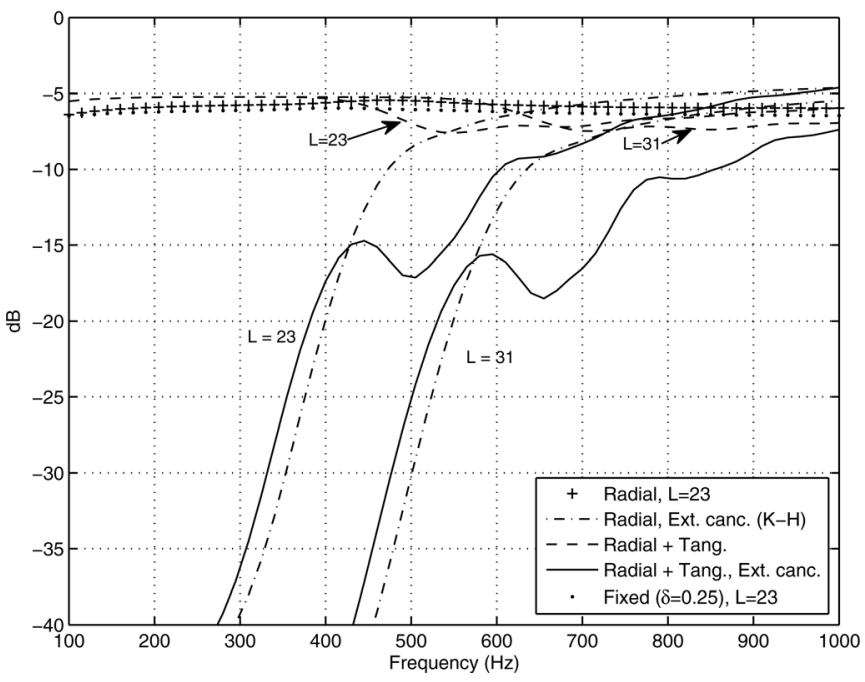

FIG. 10. Mean exterior reproduction error from $r=1.8 \mathrm{~m}$ to $r=3 \mathrm{~m}$, versus frequency, for an $L=23$ and an $L=31$ loudspeaker array, $r_{L}=1.5 \mathrm{~m}$.

mechanism for this variation is currently not well understood. Also shown in Fig. 10 are the radial plus tangential exterior field with and without exterior cancellation and the radial with exterior cancellation results for $L=31$. (The fixed and radial levels were similar to those for $L=23$ and are not shown for clarity.) The exterior cancellation is similar in form to that for the $L=23$ array but shifted in frequency as expected.

\section{CONCLUSIONS}

This paper has examined the potential for $2 \mathrm{D}$ sound reproduction systems using general first-order loudspeakers to improve accuracy and reduce the exterior sound field in comparison with simpler arrays using radially oriented fixeddirectivity or variable-directivity loudspeakers. The classical solution to the cancellation of the sound field exterior to a volume of space is the $\mathrm{K}-\mathrm{H}$ integral which assumes a continuous distribution of monopoles and dipoles normal to the surface bounding the volume. For vertical arrays of infinite extent the loudspeakers are a combination of a line source and a radial dipole line source response. Discrete arrays of these radially oriented first-order loudspeakers work well below the Nyquist frequency of the array (where the loudspeakers are half a wavelength apart) but cannot cancel the exterior field at higher frequencies. It has been shown that the addition of dipoles tangential to the surface bounding the volume can extend the cancellation of the exterior field by an octave to around twice the Nyquist frequency. It has also been shown that the interior accuracy can be improved using tangential dipoles. If the constraint of exterior cancellation is removed, the interior accuracy increase is similar to that for an array with more loudspeakers. This raises the question of whether higher accuracy can be achieved in sound reproduction using loudspeakers which have higher-order polar responses. This approach differs from other recent work which aims to increase the aliasing frequency using weighting functions ${ }^{27}$ or focusing $^{28}$ or which compensates for the directional response of loudspeakers. ${ }^{29}$ More generally, 3D arrays of loudspeakers with higher-order responses may produce reproduction errors equivalent to monopole arrays with larger numbers of loudspeakers, giving a trade-off between the number of loudspeakers and the complexity of each. We aim to consider this question for the $2 \mathrm{D}$ case in a subsequent paper.

Solutions have been derived for sound field reproduction using a continuous circular distribution of $2 \mathrm{D}$ sound sources. These solutions were then sampled for implementation using a discrete array. This approach leads to analytically tractable solutions for the loudspeaker amplitudes, but they are not applicable to discrete arrays with arbitrary geometries or to $3 \mathrm{D}$ arrays. However, solutions can also be derived for arrays with arbitrary angles and radii using a mode-matching approach. ${ }^{2,3}$ In the case of $3 \mathrm{D}$ reproduction using a spherical array of monopoles, the mode-matching solutions are similar in performance to the continuous (simple source) solution, and we would expect to see similar results in the $2 \mathrm{D}$ case. ${ }^{3}$ However, such comparisons have not been carried out here.

The results presented in this paper cannot be implemented using current commercially available loudspeaker technology, which have frequency-dependent polar responses which cannot be electronically controlled. The development of commercial loudspeakers which can provide general first-, or higher-, order polar responses will provide significant potential for improvement of sound reproduction systems.

${ }^{1}$ M. A. Gerzon, "Periphony: With-height sound reproduction," J. Audio Eng. Soc. 21(1), 2-10 (1973).

${ }^{2}$ D. B. Ward and T. D. Abhayapala, "Reproduction of a plane-wave sound field using an array of loudspeakers," IEEE Trans. Speech Audio Process. 9(6), 697-707 (2001).

${ }^{3}$ M. A. Poletti, "Three dimensional surround sound systems based on spherical harmonics," J. Audio Eng. Soc. 53(11), 1004-1025 (2005).

${ }^{4}$ O. Kirkeby and P. A. Nelson, "Reproduction of plane wave sound fields," J. Acous. Soc. Am. 94(5), 2992-3000 (1993).

${ }^{5}$ J. Daniel, "Spatial sound encoding including near field effect: Introducing distance coding filters and a viable new ambisonics format," in the AES 23rd International Conference, Copenhagen, Denmark, (May 23-25, 2003).

${ }^{6} \mathrm{~J}$. Ahrens and S. Spors, "An analytical approach to sound field reproduction using circular and spherical loudspeaker distributions," Acta Acust. Acust. 94, 988-999 (2008).

${ }^{7}$ M. A. Poletti, "A unified theory of horizontal holographic sound systems," J. Audio Eng. Soc. 48(12), 1155-1182 (2000).

${ }^{8}$ M. A. Poletti, F. M. Fazi, and P. A. Nelson, "Sound-field reproduction systems using fixed-directivity loudspeakers," J. Acoust. Soc. Am. 127(6), 3590-3601 (2010).

${ }^{9}$ A. J. Berkhout, D. de Vries, and P. Vogel, "Acoustic control by wave field synthesis," J. Acous. Soc. Am. 93(5), 2764-2778 (1993).

${ }^{10}$ M. M. Boone, E. N. G. Verheijen, and P. F. Van Tol, "Spatial sound-field reproduction by wave-field synthesis," J. Audio Eng. Soc. 43(12), 10031012 (1995).

${ }^{11}$ S. Takane, Y. Suzuki, and T. Sone, "A new method for global sound field reproduction based on Kirchhoff's integral equation," Acust. Acta Acust. 85, 250-257 (1999).

${ }^{12} \mathrm{~S}$. Ise, "A principle of sound field control based on the KirchhoffHelmholtz integral equation and the theory of inverse systems," Acust. Acta Acust. 85, 78-87 (1999).

${ }^{13}$ P. A. Nelson, H. Hamada, and S. J. Elliot, "Adaptive inverse filters for stereophonic sound reproduction," IEEE Trans. Signal Process. 40(7), 1621-1632 (1992).

${ }^{14} \mathrm{~T}$. Betlehem and T. Abhayapala, "Theory and design of sound field reproduction in reverberant rooms," J. Acous. Soc. Am. 117(Pt 1), 2100-2111 (2005).

${ }^{15}$ P.-A. Gauthier, A. Berry, and W. Woszczyk, "Sound-field reproduction in-room using optimal control techniques: Simulations in the frequency domain,” J. Acous. Soc. Am. 117(2), 662-678 (2005). 
${ }^{16}$ S. Spors, M. Renk, and R. Rabenstein, "Limiting effects of active room compensation using wave field synthesis," in the AES 118th Convention, Barcelona, Spain (May 28-31, 2005), preprint 6400.

${ }^{17}$ S. Spors, H. Buchner, R. Rabenstein, and W. Herbordt, “Active listening room compensation for massive multichannel sound reproduction systems using wave-domain adaptive filtering," J. Acous. Soc. Am. 122(1), 354-369 (2007).

${ }^{18}$ L. Fuster, J. J. Lopez, A. Gonzalez, and P. D. Zuccarello, "Room compensation using multichannel inverse filters for wave field synthesis systems," in the AES 118th Convention, Barcelona, Spain (May 28-31, 2005), preprint 6401.

${ }^{19}$ Y. J. Wu and T. D. Abhayapala, "Theory and design of soundfield reproduction using continuous loudspeaker concept," IEEE Trans. Audio, Speech, Lang. Process. 17(1), 107-116 (2009).

${ }^{20}$ D. R. White, "Wide-range electrostatic loudspeaker with a zero-free polar response," J. Audio Eng. Soc. 57(10), 822-831 (2010).

${ }^{21}$ E. G. Williams, Fourier Acoustics (Academic Press, San Deigo, 1999), pp. 115-125, 266.

${ }^{22} \mathrm{G}$. N. Watson, A Treatise on the Theory of Bessel Functions, 2nd ed. (Cambridge University Press, London, 1995), p. 361.
${ }^{23}$ M. A. Poletti, "Robust two-dimensional surround sound reproduction for nonuniform loudspeaker layouts," J. Audio Eng. Soc. 55 (7/8), 598-610 (2007).

${ }^{24}$ G. H. Golub and C. F. Van Loan, Matrix Computations, 3rd ed. (The Johns Hopkins University Press, Baltimore, MD, 1996), p. 271.

${ }^{25}$ A. V. Oppenheim and R. W. Schafer, Discrete-Time Signal Processing (Prentice-Hall International, Inc., Englewood Cliffs, NJ), p. 86.

${ }^{26}$ R. Kennedy, P. Sadeghi, T. D. Abhayapala, and H. Jones, "Intrinsic limits of dimensionality and richness in random multipath fields," IEEE Trans. Signal Process. 55(6), 2542-2556 (2007).

${ }^{27}$ E. Corteel, R. Pellegrini, and C. Kuhn-Rahloff, "Wave field synthesis rendering with increased aliasing frequency," in the AES 124th Convention, Amsterdam, The Netherlands (May 17-20, 2008), preprint 7362.

${ }^{28}$ S. Spors and J. Ahrens, "Local sound field synthesis by virtual secondary sources," in the 40th International Conference of the Audio Engineering Society, Tokyo (October 8-10, 2010).

${ }^{29}$ J. Ahrens and S. Spors, "Sound field reproduction employing non-omnidirectional loudspeakers," in the 126th Audio Engineering Society Convention, Berlin, Germany (May 7-10, 2009), preprint 7741. 